\title{
ACROPOLIS study: differences in symptoms and comorbidities in 472 patients with acromegaly according to the sex of patients and sources of clinical data
}

Philippe Caron, ${ }^{1}$ Thierry Brue, ${ }^{2}$ Gérald Raverot, ${ }^{3}$ Antoine Tabarin, ${ }^{4}$ Anne Cailleux, ${ }^{5}$ Brigitte Delemer, ${ }^{6}$ Peggy Pierre Renoult, ${ }^{7}$ Aude Houchard, ${ }^{8}$ Benedicte Duclos-Morlaes, ${ }^{8}$ Philippe Chanson ${ }^{9}$

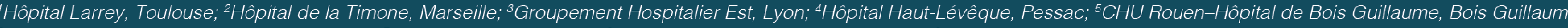

\section{Introduction}

Aromegaly is a rare, long-term, multisystem disease chacterized by excessive growth hormone (GH) (GF-1), and caused by a benign pituitary adenom A broad range of signs/symptoms and comorbidities are caused by the tumour itself and by the long-ter effects of GH/IGF-1 on multiple organs and tissues. Diagnosis is often delayed, by up to a decade in some patients. ${ }^{2}$ This reflects the non-specific nature the insidious onset of differentiating features and lack of disease awareness amongst healthcare professionals.3

As early diagnosis may increase the rate of succes reatment ${ }^{4}$ and is important for preventing long. 政 wareness of the signs/
acromegaly is key.

Objective

- The ACROPOLIS study was designed to characterize oms and comorbidities of acromegal at diagnosis in a large cohort of patients.

\section{Methods}

- Non-interventional observational cross-sectional ulticentre study conducted in France between 2013 and 2014 .

Adults with acrom

were included. from patients' medical records (transcribed into case report forms [CRFs]), and patients were also asked to complete self-administered questionnai Demographic and disease characteristics were comorbidities of acromegaly and dates of occurre A post-hoc analysis was conducted to evaluate the evolution of manifestations prior to diagnosis.

Differences in the reporting of manifestations according to data source were described usin rate of discrepancy (percentage of patients are or the patient's medical records.

\section{Results}

Patient characteristics at diagnosis of acromegaly

In total, 648 patients were enrolled in the study; of these, 472 met the inclusion criterion, had question analysis.

Patient demographic and disease characteristics are summarized in Table 1.

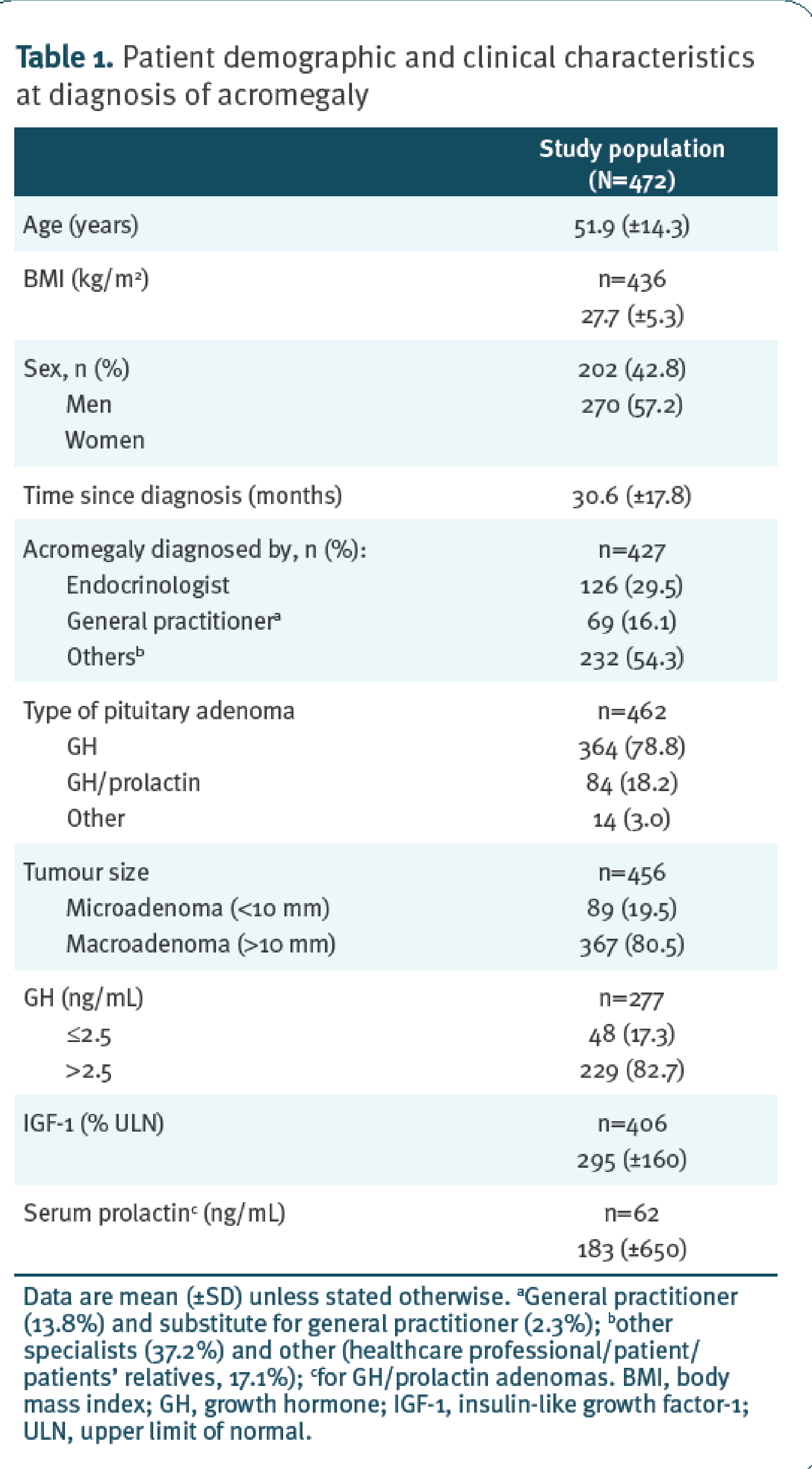

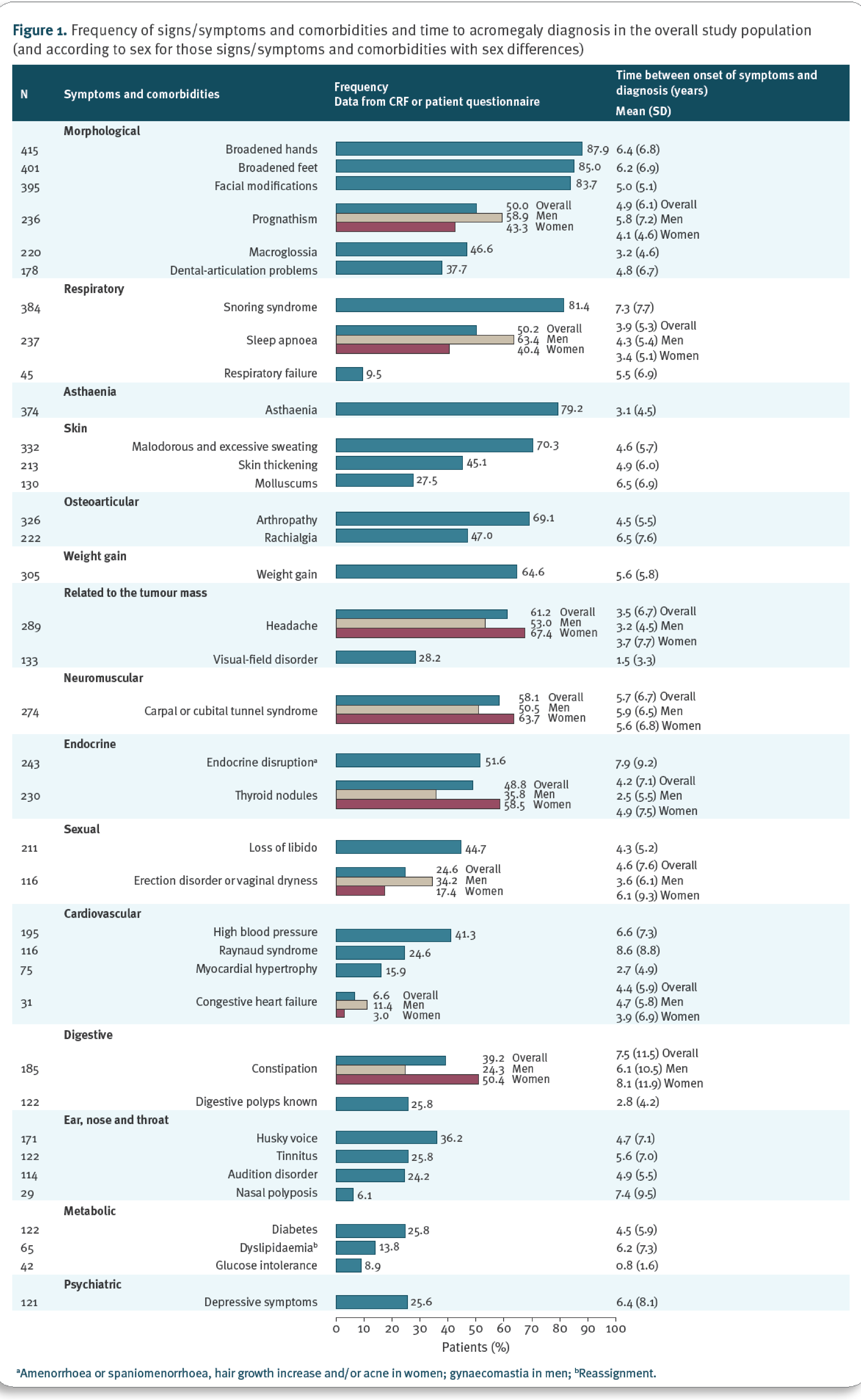

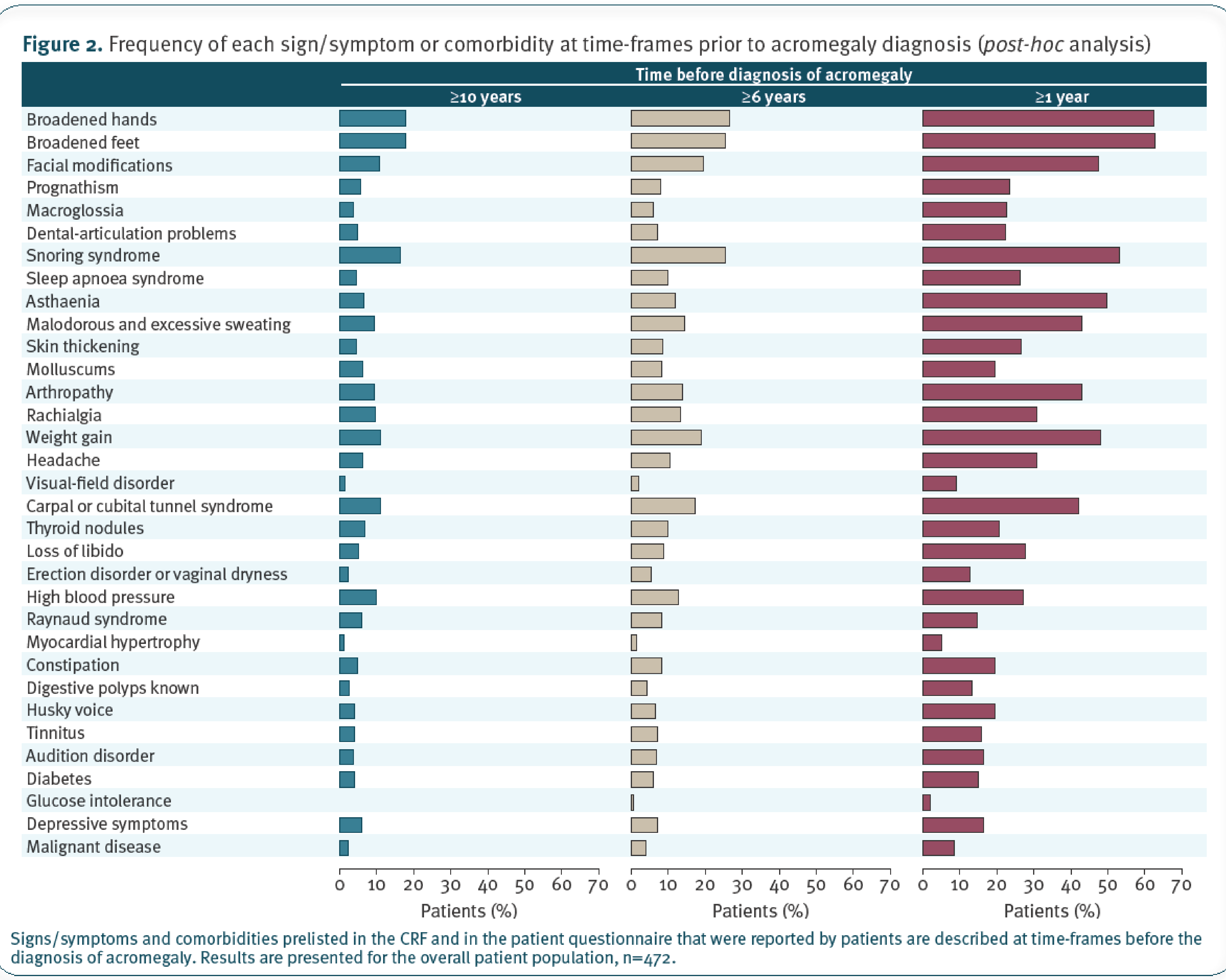

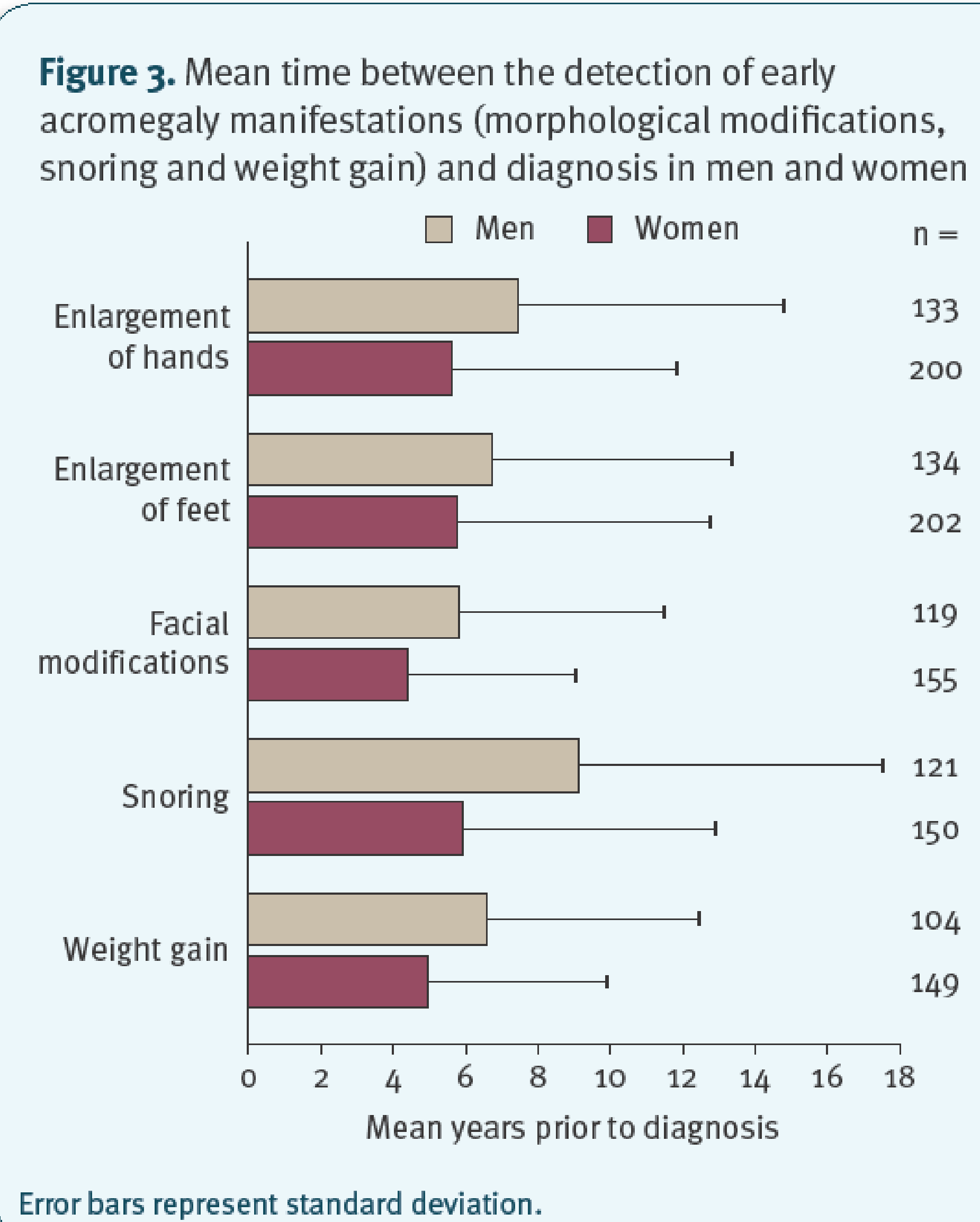

Frequency of signs/symptoms and comorbidities at diagnosis

- At diagnosis, patients presented a broad range of signs/symptoms and comorbidities.

The incidence of a number of manifestations ended to differ between the sexes: headache, carpal/cubital tunnel syndrome, constipation and thyroid nodules were more common in women, while prognathism, sleep apnoea syndrome an men (Figure 1).

Evolution of signs/symptoms and comorbidities prior to diagnosis (post-hoc analysis)

Mean (standard deviation, SD) time between onse of manifestations and acromegaly diagnosis was

The mean (SD) time between the earliest manifestation and acromegaly diagnosis was

diagnosis is summarized in Figure 2 .

The earliest manifestations were morphologic (enlargement of extremities, changes in facial features), snoring and weight gain. Morphologic manifestations, snoring and weight gain tended (Figure 3).

Differences in signs/symptoms and comorbidities according to data source

There were differences in the reporting of manifestation between the ratients' questionnaires and patients' medical records (CRFs).

Of the 39 manifestations reported, rates of discrepancy ranged from $5.5 \%$ to $36.2 \%$.

Of these, manifestations reported at highest rates of discrepancy were: snoring $(36.2 \%$ [171/472]). weight gain $(35.8 \%[169 / 472])$, loss of libido $34.5 \%[163 / 472])$, rachialgia $(33.5 \%[158 / 472])$ sthaenia (33.5\% [158/472]), and arthropathy (32.6 [154/472]).

\section{Conclusions \\ - The ACROPOLIS study confirms the broad range of acromegaly manifestation at diagnosis. \\ - Key manifestations detected earliest, prior to diagnosis, were morphological modifications, snoring and weight gain, which evolved slowly over many years. \\ - Sex differences were observed in terms of the frequency of manifestations and time between onset and diagnosis. \\ - Differences in the reporting of manifestations between patient and clinician were most evident for snoring weight gain, loss of libido, asthaenia rachialgia, and arthropathy. \\ Acknowledgements \\ study. The authors also thankWast an References Melmed S.J Clin

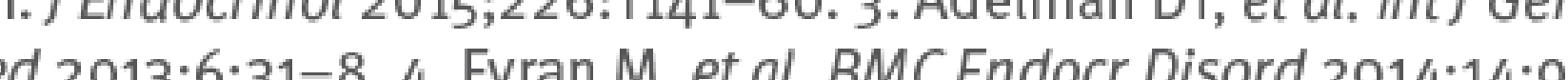
This study was sponsored by Ipsen 